Christovam Barcellos 1

Kátia Coutinho 1

Maria de Fátima Pina 1

Mônica M. A. F. Magalhães 1

Júlio C. M. D. Paola 1

Simone M. Santos 1

\section{Inter-relacionamento de dados ambientais e de saúde: análise de risco à saúde aplicada ao abastecimento de água no Rio de J aneiro utilizando Sistemas de Informações Geográficas}

\author{
Linkage of environmental and health data: \\ health risk analysis of the Rio de J aneiro water \\ supply using Geographical Information Systems
}

1 Departamento de Informações em Saúde, Centro de Informação em Ciência e Tecnologia Fundação Oswaldo Cruz. Av. Brasil 4365,

Rio de Janeiro, RJ 21045-900, Brasil. xris@dcc001.cict.fiocruz.br

\begin{abstract}
Exposureassessment of population groups is based on linkage of environmental and heal th data. This relationship can be hard to establish due to spatial and temporal lags in data sets. GIS can be used as a basis for organizing health-rel ated and environmental data sets. We examined potential health risk in the Rio de Janeiro city water supply based on the overlay of information layers containing data on the presence and quality of water supply services. We used census tracts as the primary georeferenced data, since they contain information on how households are supplied, water supply pipes, sources, and reservoirs, and water quality according to the monitoring program. Population groups exposed to risks were located and quantified using spatial operations among these layers and adopting different risk criteria. The main problems related to water supply are located on the northern slope of the Tijuca Mountain Range and in the western area of the city of Rio, where the population relies on alternative water supply sources. The different origins, objectives, and structures of data have to be analyzed critically, and GIS can be used as a data validation tool as well as an instrument for detailed identification of inconsistencies.

Key words Environment; Water Supply Systems; Environmental Analysis; Geographical Information Systems
\end{abstract}

Resumo O inter-relaci onamento de dados ambi entais e sanitários, necessário na avaliação da exposi ção de grupos populacionais a fatores de risco, é dificultado pel a defasagem no tempo e espaço destes conjuntos de dados. Neste trabal ho utilizaram-se como bases de dados georreferenciadas: os setores censitári os, que contêm informações sobre a forma com que são abastecidos os domicílios; a rede de abasteci mento de água, seus principais mananciais e reservatórios; a qualidade da água, segundo programa de monitoramento. Medianteoperações espaciais entre estas camadas, foram local izados equantificados grupos populacionais submeti dos a risco, de acordo com diferentes critérios. Grande parte dos riscos associados ao abasteci mento de água encontram-se local izados na encosta norte do Maciço da Tijuca, e na Zona Oeste, onde a população procura formas alternativas de abastecimento. Em razão das diferentes ori gens, obj eti vos e estruturas dos dados, os Sistemas de Informações Geográficas (SIG) podem ser utilizados como instrumento de organização, de vali dação destes dados e de verifi cação de possíveis inconsistências. Palavras-chave Ambiente; Abastecimento de Água; Análise Ambiental; Sistemas de Informações Geográficas 


\section{Introdução}

A identificação de grupos populacionais submetidos a risco é uma tarefa imprescindível para a elaboração de programas preventivos e como meio de avaliação de exposi ções diferenciadas. A localização destes grupos no espaço permite um maior detalhamento do contexto social e ambiental em que estas exposições ocorrem, ao mesmo tempo que introduz novas variáveis, intrínsecas ao espaço, que podem dificultar sua interpretação (Jolley et al., 1992; Barcellos \& Bastos, 1996). A investigação da relação entre fatores ambientais e efeitos sobre a saúde pressupõe uma seqüência de eventos do processo de produção de doenças representada por uma acumulação de riscos em determinados lugares delimitáveis e identificáveis no espaço. Os agravos à saúde em grupos sociais podem ser conseqüência da distribuição desigual, no espaço, de fontes de contaminação ambiental, da dispersão ou concentração de agentes de risco, da exposição da população a estes agentes e das características de suscetibilidade destes grupos (Corvalán et al., 1996). Grande parte destes determinantes à saúde são passíveis de localização no espaço. Através da união entre os processos desencadeadores de riscos ambientais, pode-se estabelecer uma complementaridade de eventos que permitem a análise globalizada de riscos à saúde (Briggs, 1992). A análise ecológica de dados ambientais e epidemiológicos pode permitir, mais que uma verificação de associações entre estes fenômenos, uma melhor compreensão do contexto em que se produzem os processos sócio-espaciais (Susser, 1994).

Os Sistemas de Informações Geográficas (SIG) têm sido utilizados como ferramenta de consolidação e análise de grandes bases de dados sobre saúde e ambiente. Estes sistemas permitem a captura, armazenamento, manipulação, análise e exibição de dados georreferenciados, isto é, relacionados a entidades gráficas com representação espacial.

O caso do abastecimento de água no Município do Rio de Janeiro pode ser utilizado como exemplo da construção de mapas de risco com informações complementares e intercambiáveis. Diversas fontes de informação podem conter dados sobre o abastecimento de água no Brasil, sendo a maior parte destes dados passíveis de georreferenciamento. Neste trabal ho, foram construídas e analisadas três camadas inter-relacionáveis de informações espaciais, envolvendo os dados do censo populacional com quesitos sobre saneamento, referidos aos setores censitários; o sistema de abastecimen- to de água, com a localização da rede principal de distribuição, mananciais e elevatórias e dados sobre a qualidade da água segundo programa de monitoramento conjunto entre o órgão de controle ambiental do estado (Feema) e a agência estadual de saneamento (Cedae), representado no mapa por pontos.

É importante destacar que cada uma destas camadas possui origem e características construtivas diferentes. O desenho da rede de distribuição, fornecido pela agência de saneamento, demonstra a capacidade de se suprir com água determinada parcela da população localizada em torno desta rede. O abastecimento efetivo destes domicílios depende, no entanto, de outras características de projeto e operação, tais como a pressão, vazão e regime de fluxo da rede, bem como a disposição desta população de pagar por este serviço. Por outro lado, os dados sobre abastecimento de água, coletados durante o censo demográfico, devem ser tomados como informações referidas pela população. Nestes questionários, a população é perguntada, entre outros itens, sobre a forma como seu domicílio é abastecido de água, se por rede pública, ou por poços e nascentes. A resposta a este item depende do entendimento dos moradores sobre a maneira preponderante como aquele domicílio é abastecido, sendo comum o abastecimento por fontes múltiplas e alternativas, principalmente em áreas de abastecimento descontinuado de água. Os dados sobre qualidade da água têm origem em programas permanentes de monitoramento, sendo imprescindível, mas não suficiente, o uso de indicadores de qualidade, que nem sempre retratam o quadro geral de riscos à saúde relacionados ao consumo de água (Batalha $\&$ Parlatore, 1977; Barcellos \& Machado, 1991). Briggs (1992) discute as possíveis formas de inter-relacionamento entre dados de fonte e receptores de risco com características geométricas diferentes. No caso da qualidade da água, estes dados, sempre referidos a pontos de amostragem, dificilmente poderiam ser extrapolados para áreas adjacentes, porque representam determinadas condições de um trecho linear da rede de distribuição. De modo geral, fenômenos ambientais podem ter três tipos de representação comuns ao geoprocessamento: o ponto (por exemplo, estações de amostragem), linha ou arco (estradas ou linhas de abastecimento) e áreas (bairros ou distritos de saneamento).

Cada uma destas informações, construídas na forma de camadas, possui, portanto, limitações de análise inerentes à sua própria construção, sendo o SIG uma ferramenta importan- 
te na integração, inter-relacionamento e validação destas informações (Briggs, 1992). Por intermédio de operações espaciais, podem-se obter informações sobre variáveis contidas em duas ou mais destas camadas, como, por exemplo, uma lista de domicílios com carência de sistemas de abastecimento de água em áreas de alta incidência de doenças relacionadas ao saneamento, ou a localização de áreas de pobreza onde problemas de saneamento são em geral agravados pela carência sócio-econômica.

A maior parte da população do Município do Rio de Janeiro (cerca de $95 \%$ dos domicílios, segundo o censo demográfico de 1991) é abastecida de água pela rede geral. Apesar disso, persistem problemas que podem ter impactos negativos sobre a saúde da população da cidade, como a contaminação da rede de distribuição de água, a precariedade ou vulnerabilidade dos sistemas de abastecimento, ou mesmo a inexistência deste serviço em algumas áreas da cidade. Por causa da conhecida heterogeneidade na ocupação do solo urbano e à acidentada topografia da cidade, é possível que os problemas com o abastecimento de água estejam concentrados em áreas e grupos sócio-espaciais característicos. O planejamento de ações de saneamento e de vigilância à saúde deve centrar-se nestas áreas, identificadas através de critérios de risco espaciais, ambientais e sócio-demográficos, que são localizados e quantificados utilizando o SIG como ferramenta de organização e análise de dados espaciais. Em adição, é descrita a construção das camadas de informações sobre o abastecimento de água no Município do Rio de Janeiro, realizada pelo projeto SIG-Fiocruz.

\section{Materiais e métodos}

Foram construídas al gumas camadas de informação que contêm dados relevantes para a caracterização de riscos relacionados ao abastecimento de água em microáreas. Nos sistemas de geoprocessamento, os dados são armazenados em forma de camadas de informação, em que cada camada corresponde a um tema, possibilitando sua manipulação isoladamente ou através de operações de análise espacial, estabelecendo relações de dados de diferentes naturezas (Câmara et al., 1995). Foi utilizada a técnica de regiões de influência (buffers) para estabelecer áreas em torno de fatores de risco, localizáveis no espaço. Esta técnica tem sido utilizada na avaliação de saúde para estimar e identificar populações expostas a riscos ambientais, como, por exemplo, a exposição a campos el etromagnéticos gerados pela transmissão de energia (Wartenberg et al., 1993). A escolha das distâncias a partir das quais são delimitadas áreas de influência (buffers) é em geral arbitrária. No presente estudo, optou-se por caracterizar estas áreas de influência tendo como base critérios teóricos, sem considerar a resposta estatística dos pontos de corte.

\section{Camada de setores censitários}

Os contornos de cerca de 6.400 setores censitários (SC) do Município do Rio de Janeiro foram transcritos visual mente da base cartográfica do IBGE, para plantas na escala 1:5000 obtidas junto à Light. Maiores detalhes sobre a construção da malha de setores censitários encontram-se em Pina (1995). A aquisição envolveu as seguintes etapas de trabal ho: correção dos contornos através de consulta ao IBGE; digitalização dos contornos utilizando software do tipo CAD; importação e edição dos contornos de SC para ambiente ARC/Info e posterior exportação para o formato Mapinfo, mantendose como indexador de dados os códigos de setores, fornecido pelo IBGE. A estes setores foram associados dados sobre saneamento e população contidos no censo demográfico (IBGE, 1992). A malha dos bairros foi gerada com a agregação de setores censitários e armazenada em camada específica.

\section{Camada do sistema de abastecimento de água}

Foram obtidas cópias heliográficas na escala 1:25000, cedidas pela Cedae, contendo o desenho dos sistemas de abastecimento de água, envolvendo a identificação e localização de reservatórios, estações de tratamento, el evatórias, adutoras e rede principal de distribuição. Este mapa foi digitalizado utilizando-se programa CAD, editado em ambiente ARC/Info e exportado para o formato Mapinfo.

\section{Camada de qualidade da água na rede de distribuição}

Foram obtidos junto à Cedae os endereços de cerca de quinhentos postos de coleta de amostras de água na forma de pontos, aos quais foram associados cerca de 12.000 dados, relativos a coletas realizadas entre 1991 e 1992, sobre a concentração de cloro livre, pH, fluoreto, cor eturbidez, bem como sobre a presença/ ausência de coliformes totais e fecais nessas amostras. O georreferenciamento dos postos (Figura 1) foi obtido pela localização e mediante ende- 
Presença de flúor na água nos postos de monitoramento da rede de distribuição de água. A área demarcada representa a região de mata atlântica da cidade, onde se concentram pequenos manaciais de água.

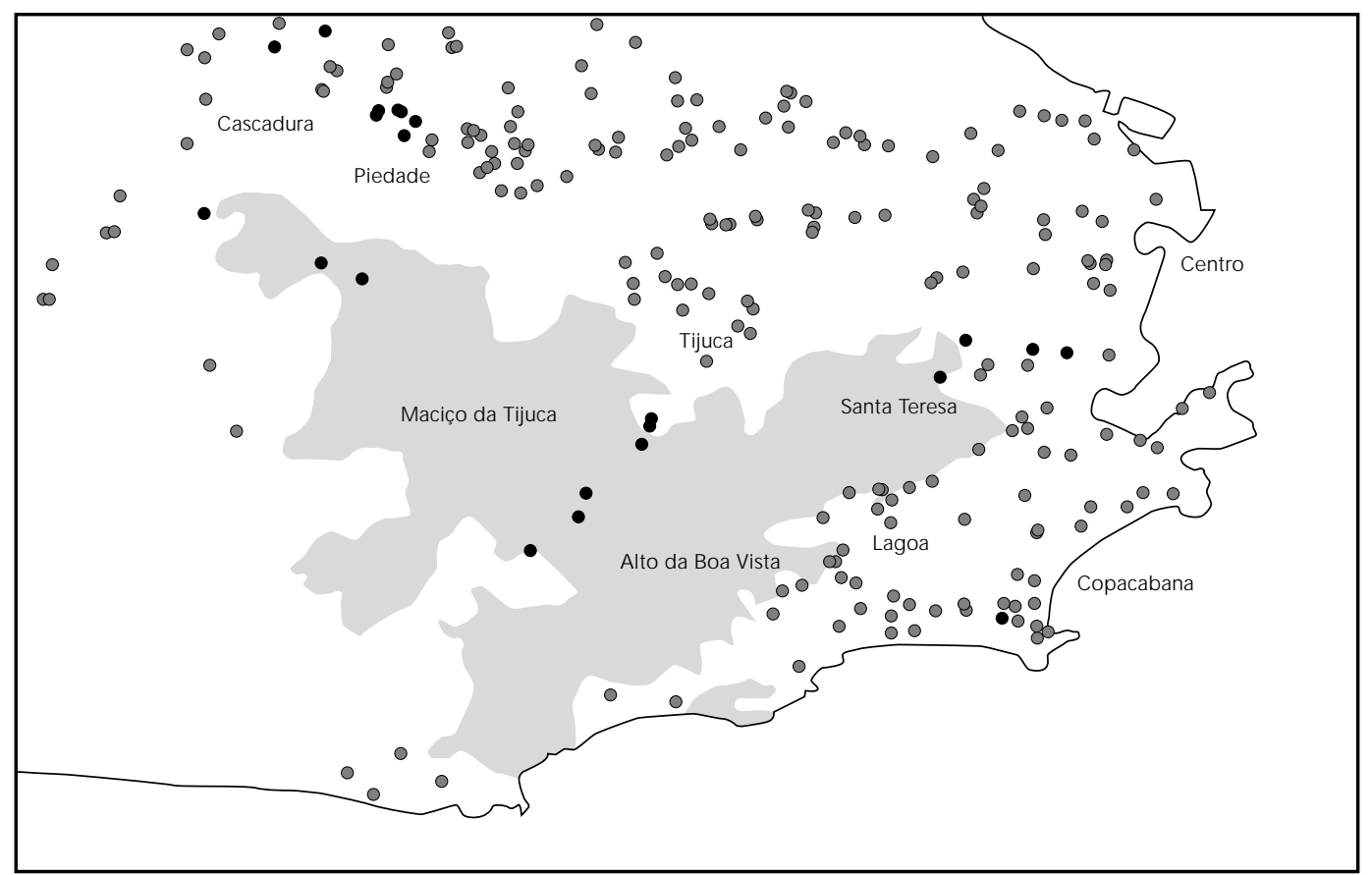

Concentração de flúor na água $(\mathrm{mg} / \mathrm{l})$

menor que 0,4

maior que 0,4

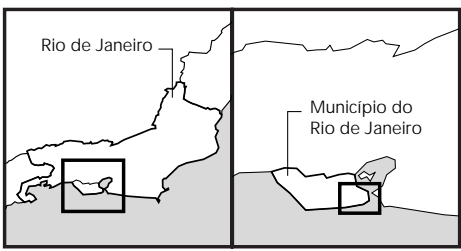

reço dos 430 postos permanentes de monitoramento em cartas em escala 1:2000 da Light, a partir das quais foram obtidos os pares de coordenadas (UTM) para cada ponto, que foram armazenadas junto ao banco de dados sobre qualidade da água.

\section{Georreferenciamento de dados ambientais e epidemiológicos}

No geoprocessamento, o trabalho com as bases de dados exige que estes estejam relacionados a unidades espaciais. Dentre as possíveis unidades espaciais de referência para dados ambientais e sanitários encontram-se o setor censitário, o território do código de endereçamento postal (CEP), o bairro, a bacia hidrográfica, o distrito sanitário, o distrito administrati- vo (subdivisão do município) e o município. Alguns critérios geográficos, estatísticos e geométricos devem ser estabelecidos para a escoIha de uma unidade mínima de agregação de dados (Barcellos e Santos, 1996). A vantagem do uso de unidades territoriais de agregação de dados é a possibilidade de se obterem denominadores para a construção de taxas utilizadas como indicadores epidemiológicos. Diversos trabalhos têm sugerido indicadores para o saneamento, escolhidos em razão de sua magnitude e importância para a saúde pública, da capacidade de resposta a alterações das condições de saneamento, bem como da validade e confiabilidade dos dados (Heller, 1997). Entre estes indicadores, a taxa de mortalidade infantil tem sido tradicionalmente utilizada para avaliar impactos de ações de saneamento sobre a saúde, sendo passível de georreferencia- 
mento no nível de bairro. A flutuação aleatória dos dados, causada pela pequena magnitude, tanto do numerador, quanto do denominador, pode inviabilizar sua utilização nesta escala (Campos, 1997).

Uma segunda estratégia de georreferenciamento de dados epidemiológicos permite a localização de eventos tendo como referência o seu endereço, gerando mapas que formam nuvens de pontos, à semelhança do famoso mapa de John Snow na investigação do surto de cólera em Londres. Neste caso, podem ser verificados estatística e visual mente aglomerados de pontos que sugerem a existência de fontes comuns de risco (Nobre \& Carvalho, 1994).

Freqüentemente, os dados de saú de e ambiente são referidos a unidades espaciais não coincidentes. O nível mínimo de agregação de dados de saneamento é o território das agências locais de água e esgoto, muitas vezes delimitado por sub-bacias hidrográficas. Dados de monitoramento de qualidade do ar e água referem-se a pontos de amostragem cujas regiões de influência são dificilmente conhecidas. Os sistemas de informações de saúde, como, por exemplo, o de mortalidade (SIM), permitem a agregação de dados por município de residência ou ocorrência do óbito, exigindo um grande esforço para o endereçamento de informações em unidades espaciais menores. No Município do Rio de Janeiro, alguns dados epidemiológicos vêm sendo apurados ou recuperados mantendo-se o bairro como campo de endereço nos sistemas de informação, o que permite seu georreferenciamento a esta unidade espacial. O uso de uma ou outra unidade espacial de referência condiciona a escala de análise dos fenômenos ambientais e epidemiológicos, alterando os resultados estatísticos e visuais destas associações (Barcellos \& Bastos, 1996).

\section{Identificação de fatores e áreas de risco}

As qualidade e quantidade de água servida à população podem ter impactos diferenciados sobre a saúde, estando muitas vezes relacionados. Por exemplo, a irregularidade do abastecimento de água de uma determinada área urbana pode permitir a introdução de agentes patogênicos na rede de distribuição de água.

Foram consideradas como fatores de risco à saúde a dificuldade na obtenção de água pela ausência de rede de distribuição nas proximidades dos domicílios, a contaminação recorrente da água por bactérias do grupo coliforme, bem como a captação de água em manan- ciais locais, sem tratamento ou sujeitos a contaminação eventual. Foram identificadas áreas e populações submetidas a risco utilizando-se os seguintes critérios ambientais e sócio-demográficos:

1) Áreas próximas a postos de monitoramento de qualidade da água que apresentam alta recorrência de contaminação por coliformes fecais. Estas áreas foram identificadas pelo desenho de áreas de influência (buffers) de 1 $\mathrm{km}$ em torno dos pontos onde mais de $20 \%$ das amostras apresentaram presença de coliformes fecais. Esta freqüência de contaminação pode indicar um severo comprometimento de trechos da rede de distribuição. A distância de influência foi estipulada com base na expectativa de sobrevivência de agentes patogênicos na água, mesmo com a presença de cloro livre (Battal ha \& Parlatore, 1977). A proximidade dos domicílios de pontos de monitoramento com contaminação recorrente pode representar risco à saúde pela possibilidade de veiculação através da rede de microorganismos patogênicos.

2) Áreas abastecidas por rede geral com predominância de águas de origem local, isto é, tomadas de pequenos mananciais localizados principalmente nos maciços da Tijuca e da Pedra Branca. Como conseqüência da crescente ocupação destas encostas é possível que haja contaminação, mesmo que esporádica, das águas por dejetos humanos. As áreas foram identificadas por meio de buffers de $2 \mathrm{~km}$ em torno dos pequenos mananciais. Por causa da topografia da cidade é esperado que águas destas encostas possam atingir grandes distâncias através da rede de distribuição. A análise dos parâmetros de qualidade da água permitiu identificar áreas afastadas que, ainda assim, sofrem influência destes mananciais (Barcellos, não publicado).

3) Áreas distantes da rede geral de distribuição, onde a rede secundária de abastecimento pode ser ausente ou apresentar problemas de continuidade de abastecimento. Estas áreas foram localizadas por meio de buffers de 0,5 km em torno dos arcos que representam a rede principal de distribuição. A quantidade de água consumida por uma população pode variar em até uma ordem de grandeza, dependendo de sua disponibilidade. O fornecimento de água de boa qualidade na fonte de abastecimento tem pouco impacto sobre as condições de saúde se o meio de coleta e transporte for manual ou inadequado (Drangert \& Lunguist, 1990). A distância dos domicílios em relação à fonte de abastecimento condiciona, portanto, a possibilidade de sua ligação através de ramais se- 
cundários, sendo uma variável importante para a garantia da qualidade do serviço.

4) Áreas onde mais de $50 \%$ dos residentes, segundo o censo de 1991, declararam não ser supridos por rede geral de abastecimento de água. Foram identificados pela base de dados os SC que correspondiam a este critério. Considerando esta variável como um somatório de respostas dos domicílios ao quesito sobre abastecimento de água durante o recenseamento pode-se presumir que, ao negar a ligação de grande parte dos domicílios à rede geral, os moradores podem apontar problemas gerais no abastecimento de água que envolvam a região, e não seu domicílio isoladamente. Dentre estes problemas podem estar incluídas a descontinuidade do abastecimento, a má qualidade da água ou a oferta local de fontes alternativas de captação.

A Tabela 1 identifica as populações e áreas submetidas a risco segundo os quatro critérios citados.

Os critérios utilizados permitiram a identificação de grupos sócio-espaciais sujeitos a riscos à saúde associados ao abastecimento de água. A contaminação da rede geral de abastecimento de água por coliformes (critério 1 ) abrange a maior parte da população sob risco, representando cerca de $35 \%$ da população total do município. $\mathrm{O}$ abastecimento de água por meio de pequenos mananciais locais (critério 2), ou a ausência de rede geral de distribuição (critério 3) podem representar riscos para parcelas ainda significativas da população da cidade (cerca de $10 \%$ ), que se encontram em regiões de mais baixa densidade populacional. Uma pequena parcela da população (cerca de $2 \%$ ) local iza-se em regiões onde a maior parte do abastecimento é real izado por fontes alternativas de água (poços e nascentes não explo- radas pela agência de saneamento), mas que representam área considerável do município (cerca de 16\% do território). Estas últimas áreas da cidade apresentam baixa densidade populacional, o que permite o uso de poços (no caso do Recreio dos Bandeirantes, Guaratiba e Sepetiba) ou nascentes (principalmente nas áreas altas do Maciço da Tijuca) para a obtenção de água.

Estes grupos sócio-espaciais, identificados segundo os diferentes critérios, são muitas vezes coincidentes, acumulando problemas como a má qualidade da água e a carência de redes de distribuição. Cerca de 375.000 habitantes do Município do Rio de Janeiro residem em áreas próximas a mananciais locais de água e com contaminação recorrente das águas da rede de distribuição, apresentando uma possível sobreposição de fatores de risco à saúde. Este é o caso do Alto da Boa Vista e áreas al tas da Tijuca, na encosta norte do Maciço da Tijuca, onde convivem bairros ricos e favelas. Outras regiões selecionadas por estes critérios possuem poucas alternativas de abastecimento, senão a rede geral de distribuição, que apresenta contaminação recorrente da água por coliformes fecais. Este é o caso de parte de Santa Teresa, Urca e Sepetiba, onde são freqüentes as queixas da população em relação ao serviço de abastecimento.

A concentração de flúor na água pode demonstrar a influência de águas provenientes de mananciais locais na rede geral de distribuição, já que estes não dispõem deste tipo de tratamento. Áreas com menor concentração de flúor (Alto da Boa Vista, Piedade e Santa Teresa) apresentam maior participação de pequenos mananciais na água distribuída à população (Figura 1), o que pode representar uma vulnerabilidade dos sistemas de abastecimento quanto à

Tabela 1

Localização, população residente e área de risco segundo critérios de qualidade do abastecimento de água.

\begin{tabular}{|c|c|c|c|}
\hline Critério de risco & $\begin{array}{c}\text { População } \\
\text { (n de residentes) }\end{array}$ & Área $\left(\mathrm{km}^{2}\right)$ & Localização \\
\hline Contaminação da água & 1.900 .000 & 349 & $\begin{array}{l}\text { Encostas das Zonas Sul e Norte, } \\
\text { parte da Zona O este }\end{array}$ \\
\hline Uso de pequenos mananciais & 700.000 & 392 & $\begin{array}{l}\text { Em torno dos maciços da Tijuca } \\
\text { e Pedra Branca }\end{array}$ \\
\hline Ausência de rede & 600.000 & 156 & $\begin{array}{l}\text { Zona O este, Barra da Tijuca } \\
\text { e áreas isoladas da Zona Norte }\end{array}$ \\
\hline Uso de fontes alternativas de água & 90.000 & 206 & $\begin{array}{l}\text { Zona O este, Maciço da Pedra } \\
\text { Branca e Alto da Boa Vista }\end{array}$ \\
\hline
\end{tabular}


contaminação dos mananciais. Nestas áreas, diversos domicílios utilizam poços e nascentes como forma de abastecimento de água, segundo o censo de 1991. Esta alternativa vem sendo adotada por grande parte dos moradores das encostas do Maciço da Tijuca, onde existem algumas fontes locais de captação de água, sem tratamento para desinfecção e fluoretação. Estes pequenos sistemas são muitas vezes custeados pelos moradores locais.

A Figura 2 mostra a superposição das camadas de informação que representam a rede geral de abastecimento e a proporção da população que declarou no censo de 1991 ser suprida pela mesma rede. Ambas informações tratam de um mesmo tema, tendo, no entanto, di- ferentes significados por causa do instrumento de aferição utilizado. A comparação entre estas duas camadas permite a validação dos dados sobre abastecimento de água na cidade. Ambos desenhos cobrem, principalmente, a mancha urbana do município, observando-se a carência na cobertura da rede principal nas áreas altas e de baixa densidade populacional, como os maciços da Pedra Branca e Tijuca. Segundo o mapa, podem-se observar áreas potencialmente cobertas pelo serviço, isto é, próximas à rede de distribuição de água, onde, no entanto, grande parte da população residente declara ser suprida por outras fontes de água, independentes da agência de saneamento. Estas áreas situam-se na Zona Oeste, Serra do Mendanha e

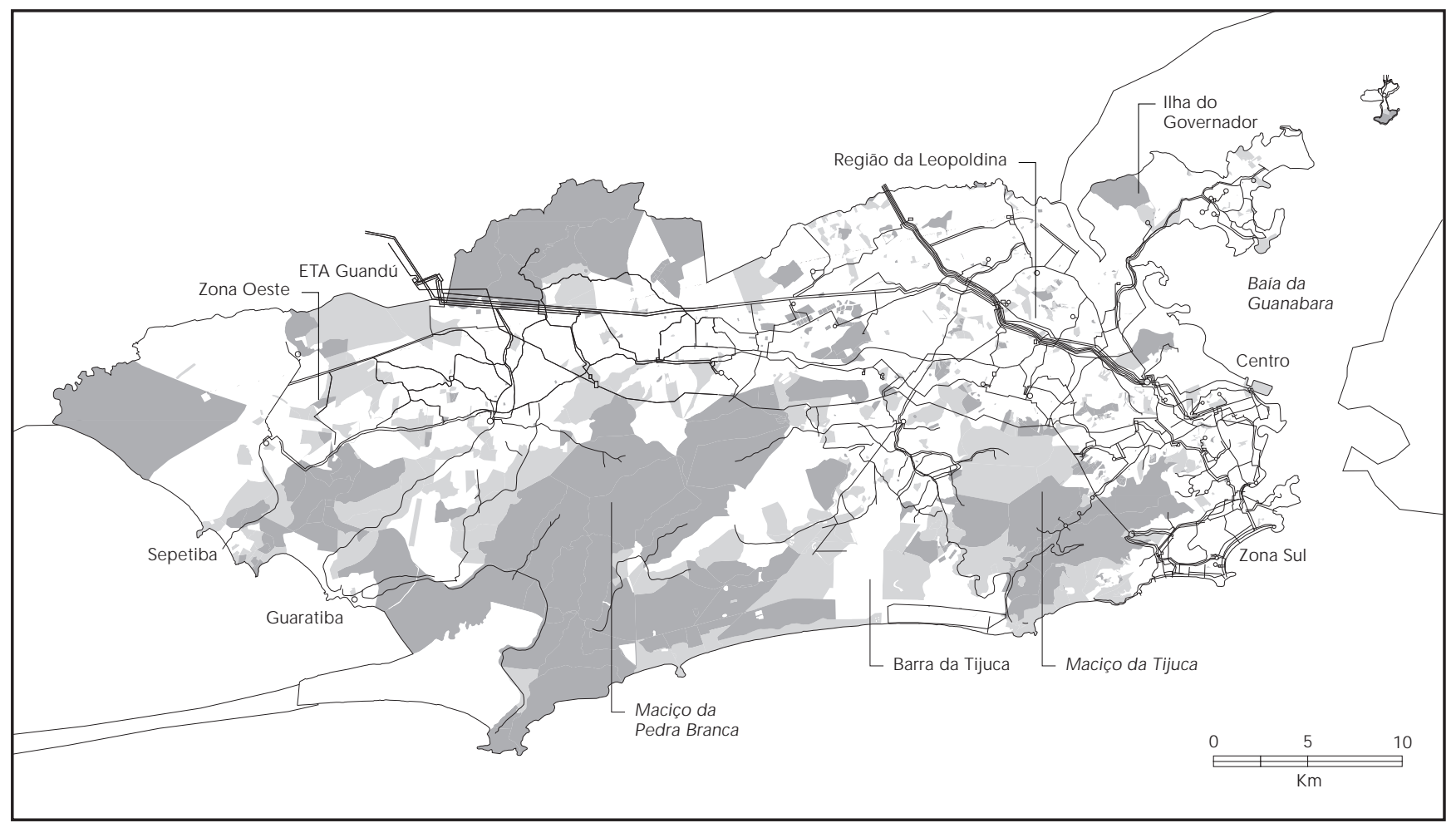

Cobertura da rede de abastecimento de água

menor que $95 \%$

75 a $95 \%$

menor que $75 \%$

__ Rede principal de distribuição de água

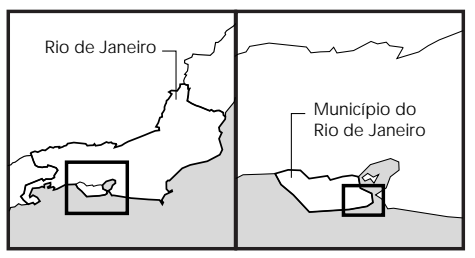


Alto da Boa Vista. Neste caso, maiores investigações devem ser realizadas localmente para esclarecer a forma de abastecimento de água e as causas da recusa (por parte da população) ou do mal funcionamento (por parte da agência de saneamento) que levaram a esta aparente contradição entre informações.

\section{Considerações finais}

Durante as últimas décadas, observou-se um incremento nos serviços de saneamento no Brasil, principalmente efetuado pelo abastecimento de água em regiões metropolitanas. A cidade do Rio de Janeiro, por exemplo, possui apenas 5\% de domicílios não abastecidos pela rede geral de distribuição. Vários problemas, contudo, ainda são encontrados no que tange aos aspectos qualitativos e quantitativos do abastecimento de água. Uma parcela considerável da população é abastecida com águas eventual mente contaminadas, utiliza-se de fontes alternativas para o abastecimento de água ou encontra-se em áreas com regime deficiente de abastecimento. Estes grupos, muitas vezes apontados genericamente como carentes ou pobres, são socialmente identificáveis e espacialmente delimitáveis.

Nesse trabalho foi possível observar uma superposição de fatores que poderiam explicar a ausência de ligações de domicílios à rede geral em áreas da cidade submetidas simultaneamente à baixa qualidade do serviço e localização em áreas de pequena urbanização, presentes em áreas faveladas, mas também em áreas de bairros nobres onde as condições sócioeconômicas são melhores.
A qualidade da água de uma rede de distribuição está fortemente relacionada ao seu regime de distribuição e características de projeto e operação do sistema de abastecimento (Clark \& Cyle, 1990). Variações de pressão interna da canalização podem facilitar vazamentos e infiltrações e provocar sua contaminação. A qualidade da água servida à população está, portanto, relacionada à sua quantidade. Alguns dos indicadores relacionados neste trabal ho, como a presença de coliformes e a concentração de cloro livre, podem exprimir ambos problemas de abastecimento.

Utilizando-se o SIG pode-se sobrepor informações sócio-ambientais que permitam uma melhor focalização destes grupos e o planejamento de ações de saneamento e vigilância à saúde. Os critérios utilizados neste trabalho podem ser aplicados e aperfeiçoados a fim de melhor identificar áreas e grupos de risco em microáreas. Além disso, o SIG, como outros métodos de mapeamento podem ser utilizados como instrumento didático e de debate com a população leiga sobre suas condições e inserção no espaço urbano (Sparrow, 1992).

Os SIGs podem manipular bases de dados que têm diferentes expressões geométricas e lógicas. Esta heterogeneidade está presente em todas as etapas de desenvolvimento do SIG: a adoção do modelo conceitual, formato de dados, software, hardware e linguagem (Strauch et al., 1996). Esta característica permite uma interoperabilidade de dados, impossível em sistemas de informação convencionais, isto é, tabulares. Por outro lado, exige que, já na fase de construção do SIG, sejam previstas as possíveis operações entre camadas de dados e sua forma de armazenamento. 


\section{Referências}

BARCELLOS, C. \& MACHADO, J. H., 1991. Seleção de indicadores epidemiológicos para o saneamento. BIO, out/dez:37-41

BARCELLOS, C. \& BASTOS, F. I., 1996. Geoprocessamento ambiente e saúde: uma união possível? Cadernos de Saúde Pública, 12:389-397.

BARCELLOS, C. \& SANTOS, S. M., 1996. Georreferenciamento de dados secundários sobre ambiente e saúde. I Semana Estadual de Geoprocessamento 10 Segeo. Rio de Janeiro: Forum Estadual de Geoprocessamento.

BATTALHA, B. H. L. \& PARLATORE, A. C., 1977. Controle da Qualidade da Água para Consumo Humano. Bases Conceituais e Operacionais. São Paulo: Cetesb.

BRIGGS, D. J., 1992. Mapping Environmental Exposure. In: Geographical and Environmental Epidemiology: Methods for Small-Area Studies (P. EIliot, J. Cuzick, D. English \& R. Stern, eds.), pp. 158176, Tokyo: Oxford University Press.

CÂM ARA, G.; FREITAS, U.; CASANOVA, M. A., 1995. Fields and objects algebras for GIS operations. III Simpósio Brasileiro de Geoprocessamento. São Paulo.

CAM POS, T. P., 1997. Perfil de Nascimentos e Óbitos Infantis: A Busca da Assistência no Município do Rio de Janeiro. Dissertação de Mestrado, Rio de Janeiro: Escola Nacional de Saúde Pública, Fundação Oswaldo Cruz.

CLARK, R. M. \& CYLE, J. A., 1990. Measuring and modeling variations in distribution system water quality. Journal of American Water Works Association, 82:46-52.

CORVALAN, C.; BRIGGS, D. \& KJELLSTROM , T., 1996. Development of environmental health indicators. In: Linkage Methods for Environment and Health Analysis (D. Briggs, C. Corvalán \& M. Nurminen, eds.), pp. 19-53, Genebra: OMS.
DRANGERT, J. O. \& LUNDGUIST, J., 1990. Household water and health: Issues of quality, quantity, handling and costs. In: Society, Environment and Health in Low-Income Countries (E. Norberg \& D. Finer, eds.), pp. 71-86, Goteborg: Karolinska Institutet.

JOLLEY, D. J.; JARMAN, B. \& ELLIOT, P., 1992. Socioeconomic confounding. In: Geographical and Environmental Epidemiology: Methods for SmallArea Studies (P. Elliot, J. Cuzick, D. English \& R. Stern, eds.), pp. 158-176, Tokyo: Oxford University Press.

HELLER, L., 1997. Saneamento e Saúde. Brasília: Organização Panamericana da Saúde.

IBGE (Instituto Brasileiro de Geografia e Estatística), 1992. Censo Demográfico de 1991. Rio de Janeiro: Fundação Instituto Brasileiro de Geografia e Estatística.

NOBRE, F. F. \& CARVALHO, M. S., 1994. Spatial and temporal analysis of epidemiological data. GIS for Health and the Environment. Sri Lanka: Colombo.

PINA, M. F., 1995. A implementação de um sistema de informações geográficas para estudos na área de saúde. XVI Congresso Brasileiro de Cartografia, Salvador.

SPARROW, M. K., 1992. From data warehouse to information craft shop: the changing shape of information support for environmental protection. Environment International, 18:3-9.

STRAUCH, J. C.; MATTOSO, M. L. \& SOUZA, J. M., 1996. Interoperabilidade de bases de dados espaciais heterogêneas e distribuídas. I Semana Estadual de Geoprocessamento, Rio de Janeiro.

SUSSER, M., 1994. The logic in ecological: II. The logic of design. American Journal of Public Health, 84:831-835.

WARTENBERG, D.; GREENBERG, M. \& LATHROP, R., 1993. Identification and characterization of populations living near high-voltage transmission lines: a pilot study. Environmental Health Perspectives, 101:626-632. 